\title{
Maximal orness weights with a fixed variability for OWA operators
}

\author{
T. Marchant \\ Ghent University \\ thierry.marchant@UGent.be
}

January 17, 2006

\begin{abstract}
When using the ordered weighted average operator, it can happen that one wants to optimize the variability (measured by the entropy (maximal) or by the variance (minimal)) of the weights while keeping the orness of this operator at a fixed level. This has been considered by several authors. Dually, there might be some contexts where one wishes to maximize the orness while guaranteeing some fixed variability. In this paper, we present two algorithms for finding such weights, when the variability is captured by the entropy and by the variance.
\end{abstract}

Keywords: OWA, orness, entropy, variability

\section{Introduction}

Anyone willing to use the ordered weighted average operator (OWA) must at some point choose a weight vector. Several approaches exist for this. For instance, [5] suggested that, in some cases, it might be interesting to choose the weight vector maximizing the entropy of the operator while guaranteeing some predetermined orness. He also proposed a technique achieving this goal. [1] studied the same problem. [3] followed the same approach and suggested a different algorithm for the maximal-entropy weight vector given a fixed orness. Since entropy is not the only possible measure for the variability, [4] presented an algorithm for finding the minimal-variance weight vector given a fixed orness.

Consider now the following situation: we are facing an aggregation problem and, after discussion with the decision-maker or for some theoretical reasons, we want to use a disjunctive operator. If we use the maximum operator, then we take into account only the largest score. So, if we aggregate $n$ scores, we use only $1 / n$ of the available information. This is quite low. It might therefore be interesting to use an 'as disjunctive as possible' operator while using at least $50 \%$ of the available information. This is possible if we use an OWA operator. We can try to find the weight vector maximizing the orness of the OWA operator 
while guaranteeing that a quantity $\beta$ of the available information (measured by the entropy) will be used in the aggregation.

Remark that this problem is dual to the one described in the first paragraph: given a fixed orness, which weight vector maximizes the entropy?

In this paper, we present an algorithm for finding weights maximizing the orness, given a fixed entropy. Since entropy is a measure of dispersion or variability and since variance is also a measure of variability, we also present an algorithm for finding weights maximizing the orness, given a fixed variance. Finally note that $[6]$ have solved a similar problem: finding the weight vector maximizing the orness, given a fixed dispersion measured by

$$
\frac{1}{n-1} \frac{1-\bar{w}}{\bar{w}}
$$

where $\bar{w}$ is the largest weight in the vector.

\section{Fixed entropy}

If $W=\left(w_{1}, \ldots, w_{n}\right)^{T}$ is the weighting vector of an OWA operator, then the orness of the operator is defined by

$$
\operatorname{orness}(W)=\frac{1}{n-1} \sum_{i=1}^{n}(n-i) w_{i}
$$

and its dispersion or entropy, by

$$
\operatorname{disp}(W)=-\sum_{i=1}^{n} w_{i} \ln w_{i}
$$

Suppose we want to maximize the orness and respect the constraint that the entropy must be equal to some given positive value $\beta$. If $\beta$ is equal to the highest possible entropy, i.e. $\beta=\bar{\beta}=\ln n$, then the solution is simply $W=$ $(1 / n, \ldots, 1 / n)^{T}$. If $\beta<\bar{\beta}$, then we can use the method of Lagrange multipliers.

Let

$$
L\left(W, \lambda_{1}, \lambda_{2}\right)=\frac{1}{n-1} \sum_{i=1}^{n}(n-i) w_{i}+\lambda_{1}\left(-\sum_{i=1}^{n} w_{i} \ln w_{i}-\beta\right)+\lambda_{2}\left(\sum_{i=1}^{n} w_{i}-1\right) .
$$

The partial derivatives of $L$ w.r.t. $w_{j}$ are

$$
\frac{\partial L}{\partial w_{j}}=\frac{n-j}{n-1}-\lambda_{1}\left(\ln w_{j}+1\right)+\lambda_{2}=0, \forall j .
$$

If we set $j$ equal to $n$, we obtain

$$
\lambda_{2}=\lambda_{1}\left(\ln w_{n}+1\right) .
$$


Replacing in (1) and letting $j=1$, we find

$$
\lambda_{1}=\frac{1}{\ln w_{1} / w_{n}} .
$$

We can now replace $\lambda_{1}$ and $\lambda_{2}$ in (1), and, after some simple algebra, we find

$$
\frac{w_{j}}{w_{n}}=\left(\frac{w_{1}}{w_{n}}\right)^{n-j / n-1}, 1 \leq j \leq n .
$$

Let us now compute the ratio of two consecutive weights:

$$
\frac{w_{j+1}}{w_{j}}=\left(\frac{w_{n}}{w_{1}}\right)^{1 / n-1}, 1 \leq j \leq n-1 .
$$

The ratio $\left(w_{n} / w_{1}\right)^{1 / n-1}$ is independent of $j$ and is not equal to 1 because $\beta<\bar{\beta}$. Let us denote it by $a$. Because we maximize the orness, it is clear that the weights may not increase with $i$, i.e. $w_{1} \geq w_{2} \geq \ldots \geq w_{n}$. So, $0<a<1$. We rewrite the last equation, using $a$ :

$$
w_{j}=w_{1} a^{j-1}, 1 \leq j \leq n .
$$

The sum of the weights being equal to 1 , we have

$$
1=w_{1} \sum_{i=1}^{n} a^{i-1}=w_{1} \frac{1-a^{n}}{1-a}
$$

whence

$$
w_{j}=\frac{1-a}{1-a^{n}} a^{j-1}
$$

So, if we know $a$, we can compute any weight. In order to find $a$, we rewrite the constraint on the entropy.

$$
\begin{aligned}
\beta & =-\sum_{i=1}^{n} w_{i} \ln w_{i} \\
& =-\sum_{i=1}^{n} \frac{1-a}{1-a^{n}} a^{i-1} \ln \frac{1-a}{1-a^{n}} a^{i-1}
\end{aligned}
$$

The ratio $a$ is one of the solutions of this equation, satisfying $0<a<1$. It cannot be solved analytically but numerical solutions with any given precision can easily be obtained. From the definition of our optimization problem, it is clear that a solution always exists in $] 0,1[$. We now prove that there is only one solution in $] 0,1[$. When $a$ is close to 1 , all weights are almost equal and the dispersion is very small (the entropy is large). When $a$ decreases and goes to 0 , the weights differ more an more from each other and have thus a larger dispersion (the entropy is small). It is thus clear that the right-hand part of (3) 
is strictly increasing in $a$ on $] 0,1[$ and equation (3) can have only one solution satisfying $0<a<1$.

Note that the weights solving our constrained maximization problem might be called exponential since any weight $(j=1, \ldots, n)$ is an exponential function of $j-1$ as shown in (2). The OWA operator based on this weight vector might hence be called an exponential OWA but [2] already used this name for another OWA operator which, actually, is not exactly exponential. Indeed, in [2], the weights follow an exponential function not for all $j$ but only for $j=1, \ldots, n-1$. More precisely, for some $\gamma \in[0,1]$,

$w_{1}=\gamma, w_{2}=\gamma(1-\gamma), w_{3}=\gamma(1-\gamma)^{2}, \ldots, w_{n-1}=\gamma(1-\gamma)^{n-2}, w_{n}=(1-\gamma)^{n-1}$.

\section{$3 \quad$ Fixed variance}

The variance of a weighting vector $W$ is defined by

$$
\operatorname{var}(W)=\frac{1}{n} \sum_{i=1}^{n} w_{i}^{2}-\frac{1}{n^{2}}
$$

If we want to maximize the orness and respect the constraint that the variance must be equal to some given positive value $\beta$, it can happen that some weights are zero. Of course, because the orness must be maximal, these weights are located at the end of the weighting vector. So, we can write the optimal weighting vector as $W_{\text {opt }}=\left(w_{1}, \ldots, w_{j}, \ldots, w_{p}, 0, \ldots, 0\right)^{T}$, for some $p \in\{1,2, \ldots, n\}$.

In order to find the optimum, we can use the method of Lagrange multipliers. Let

$$
\begin{aligned}
L\left(W, \lambda_{1}, \lambda_{2}, \lambda_{1}^{\prime}, \ldots, \lambda_{n-p}^{\prime}\right)= & \frac{1}{n-1} \sum_{i=1}^{p}(n-i) w_{i}+\lambda_{1}\left(\frac{1}{n} \sum_{i=1}^{p} w_{i}^{2}-\frac{1}{n^{2}}-\beta\right) \\
& +\lambda_{2}\left(\sum_{i=1}^{p} w_{i}-1\right)+\sum_{i=1}^{n-p} \lambda_{i}^{\prime} w_{n+1-i} .
\end{aligned}
$$

The multipliers $\lambda_{i}^{\prime}$ correspond to the fact that the last weights are zero.

The partial derivatives of $L$ w.r.t. $w_{j}$ are

$$
\frac{\partial L}{\partial w_{j}}= \begin{cases}\frac{n-j}{n-1}+2 \frac{\lambda_{1}}{n} w_{j}+\lambda_{2}=0, & j=1, \ldots, p, \\ \lambda_{j}^{\prime}=0, & j=p+1, \ldots, n .\end{cases}
$$

Setting successively $j=1$ and $j=p$ in (4), it is easy to find

$$
\lambda_{1}=\frac{p-1}{n-1} \frac{n}{2\left(w_{p}-w_{1}\right)}
$$

and

$$
\lambda_{2}=\frac{(n-1) w_{p}-(n-p) w_{1}}{(n-1)\left(w_{1}-w_{p}\right)} .
$$


Substitution in (4) yields

$$
\frac{n-j}{n-1}+\frac{p-1}{n-1} \frac{w_{j}}{w_{p}-w_{1}}+\frac{(n-1) w_{p}-(n-p) w_{1}}{(n-1)\left(w_{1}-w_{p}\right)}=0, j=1, \ldots, p .
$$

If we solve for $w_{j}$, we obtain

$$
w_{j}=w_{1} \frac{p-j}{p-1}+w_{p} \frac{j-1}{p-1}, j=1, \ldots, p .
$$

From the condition $\sum_{i=1}^{p} w_{i}=1$, we get

$$
w_{p}=\frac{2}{p}-w_{1}
$$

and

$$
w_{j}=w_{1} \frac{p-2 j+1}{p-1}+\frac{2}{p} \frac{j-1}{p-1} .
$$

We now rewrite the condition on the variance:

$$
\operatorname{var}(W)=\beta=\frac{1}{n} \sum_{i=1}^{n} w_{i}^{2}-\frac{1}{n^{2}}
$$

Substituting (5) in (6) and using the relation

$$
\sum_{i=1}^{p} i^{2}=\frac{1}{6} p(p+1)(2 p+1)
$$

we can rewrite (6) in the form of a polynomial in $w_{1}$ :

$$
w_{1}^{2} n p^{2}(p+1)-w_{1} 2 n p(p+1)-3 n^{2} \beta p(p-1)-3 p(p-1)+4 n p-2 n=0
$$

whose roots are

$$
w_{1}=\frac{1}{p}+\frac{\sqrt{3 n(p+1)(p-1)\left(n^{2} \beta p-n+p\right)}}{n p(p+1)}
$$

and

$$
w_{1}=\frac{1}{p}-\frac{\sqrt{3 n(p+1)(p-1)\left(n^{2} \beta p-n+p\right)}}{n p(p+1)} .
$$

But, because we want to maximize the orness, $w_{1}$ must be as large as possible. So, only the first root is the solution of our problem and we can write

$$
\begin{aligned}
w_{j}= & \left(\frac{1}{p}+\frac{\sqrt{3 n(p+1)(p-1)\left(n^{2} \beta p-n+p\right)}}{n p(p+1)}\right) \frac{p-2 j+1}{(p-1)} \\
& +\frac{2}{p} \frac{j-1}{p-1}, j=1, \ldots, p .
\end{aligned}
$$


So, we now have an explicit expression for the non-zero weights but it depends on $p$. Given $\beta$, we must find the value of $p$ that maximizes the orness while respecting two conditions: all weights must be (i) non-negative and (ii) real. We first consider (i). Because the weights $w_{j}$ decrease with $j$, we must only check that $w_{p}$ is non-negative, i.e.

$$
w_{p}=\frac{1}{p}-\frac{\sqrt{3 n(p+1)(p-1)\left(n^{2} \beta p-n+p\right)}}{n p(p+1)} \geq 0 .
$$

We obtain

$$
\beta \leq \frac{n(p+1)}{3 n^{2} p(p-1)}+\frac{1}{n p}-\frac{1}{n^{2}} .
$$

We now turn to (ii). The weights will be real if

$$
3 n(p+1)(p-1)\left(n^{2} \beta p-n+p\right) \geq 0 .
$$

Combining this and (8), we find

$$
\frac{n-p}{n^{2} p} \leq \beta \leq \frac{n(p+1)}{3 n^{2} p(p-1)}+\frac{1}{n p}-\frac{1}{n^{2}} .
$$

So, we will use for $p$ the integer maximizing the orness and such that (9) holds. Example. Let $n=5$ and $\beta=0.05$.

$$
\begin{gathered}
\frac{n-p}{n^{2} p}= \begin{cases}0.060, & p=2 \\
0.027, & p=3 \\
0.010, & p=4 \\
0.000, & p=5 .\end{cases} \\
\frac{n(p+1)}{3 n^{2} p(p-1)}+\frac{1}{n p}-\frac{1}{n^{2}}= \begin{cases}0.160, & p=2 \\
0.071, & p=3 \\
0.038, & p=4 \\
0.020, & p=5 .\end{cases}
\end{gathered}
$$

So, 3 is the only value for $p$ such that (9) holds. We then compute the weights using (7) with $p=3$ and we obtain $W_{\text {opt }}=(0.57,0.33,0.1,0,0)^{T}$.

\section{Conclusion}

We presented two algorithms for obtaining the OWA operator with maximal orness and a given variability, be it measured by the variance or the entropy. If, instead of a disjunctive operator, one wants to use a conjuctive operator, one may be willing to maximize the andness, with a given variability. In that case, a similar approach to the one presented in this paper can be followed.

\section{Acknowledgements}

This research was partially supported by the Bilateral Scientific and Technological Cooperation Flanders-Hungary BIL00/51 (B-08/2000). 


\section{References}

[1] M. Carbonell, M. Mas, and G. Mayor. On a class of monotonic extended OWA operators. In The Sixth IEEE International Conference on Fuzzy Systems, pages 1695-1700, 1997.

[2] D. Filev and R. R. Yager. On the issue of obtaining OWA operator weights. Fuzzy Sets and Systems, 94:157-169, 1998.

[3] R. Fuller and P. Majlender. An analytic approach for obtaining maximal entropy OWA operator weights. Fuzzy Sets and Systems, 124:53-57, 2001.

[4] R. Fuller and P. Majlender. On obtaining minimal variability OWA operator weights. Fuzzy Sets and Systems, 136:203-215, 2003.

[5] M. O'Hagan. Aggregating template or rule antecedents in real-time expert systems with fuzzy set logic. In 22nd Annual IEEE Asilomar Conf. on Signals, Systems, Computers, pages 681-689. Pacific Grove, 1988.

[6] L. Troiano and R. R. Yager. A meaure of dispersion for OWA operators. In G. C. Y. Liu and M. Ying, editors, Proceedings of the Eleventh International Fuzzy systems Association World Congress, Beijing. Tsinghua University Press and Springer, 2005. 